\section{Radiologischer und symptomatischer Verlauf der Hüft- und Kniegelenksarthrose}

Schiphof D et al. The clinical and radiographic course of early knee and hip osteoarthritis over 10 years in CHECK (Cohort Hip and Cohort Knee). Osteoarthritis Cartilage 2019; 27: 1491-1500

In der CHECK- Studie wurde der radiologische und symptomatische Verlauf bei Patienten mit Hüft- oder Kniebeschwerden, bei denen der Verdacht auf frühe Arthrose besteht, dokumentiert.

Die CHECK-Studie (Cohort Hip and Cohort Knee) ist eine multizentrische prospektive Kohortenstudie mit 1002 Patientinnen und Patienten mit ersten Beschwerden in den Knien und/oder Hüften, an der sich 10 Zentren in den Niederlanden beteiligen. Eingeschlossen wurden Patienten im Alter zwischen 45 und 65 Jahren, die erstmalig über Schmerzen oder Steifheit im Knie und/oder Hüfte 6 Monate vor Beginn der Studie ihren Hausarzt aufsuchten. Zu Studienbeginn und nach 2, 5, 8 und 10 Jahren erfolgten körperliche und radiologische Untersuchungen. Der klinische Befund einer Arthrose (OA) wurden nach den ACR-Kriterien (Clinical American College of Rheumatism) beurteilt, eine radiologische Arthrose lag nach einem Kellgren- und Lawrence-Score $\geq 2$ vor. Zu den Untersuchungsterminen erfolgten Selbsteinschätzungen der Studienteilnehmer mithilfe der WOMAC -Fragebögen (Western Ontario and McMaster Universities Osteoarthritis Index).

Von den 1002 Studienteilnehmern gingen im Verlauf der Nachbeobachtung 145 Personen verloren. Danach beendeten $85 \%$ der Probanden die 10-Jahres-Nachsorgephase. Die Mehrzahl der Teilnehmer war weiblich (79\%). Das mittlere Alter zu Studienbeginn betrug $56 \pm 5$ Jahre und der Body Mass Index (BMI) $26 \pm 4 \mathrm{~kg} / \mathrm{m}^{2}$. Über Knieprobleme klagten $83 \%$, über Hüftprobleme $59 \%$ und über beides $42 \%$ der Teilnehmer. Der mediane WOMAC-Score betrug 25 \pm 16,0 und der Wert für Schmerzen in Knie und/Hüfte über die vergangene Woche erreichte

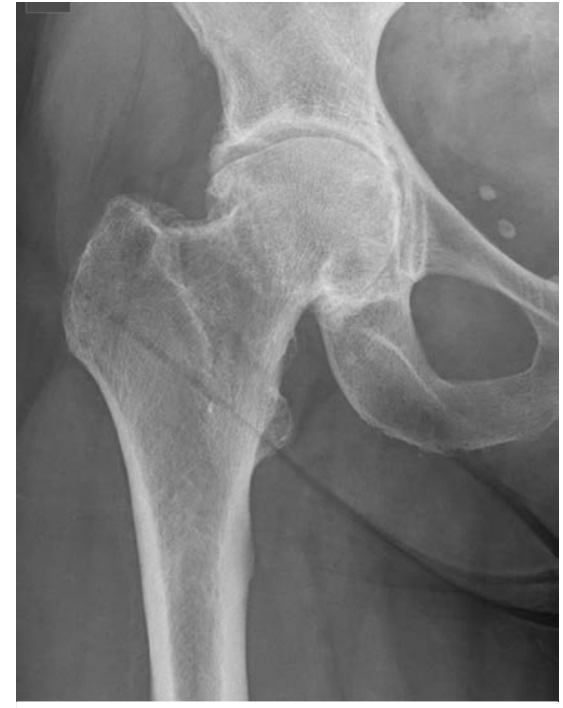

Ausgeprägte Protrusionskoxarthrose: Der deformierte Hüftkopf wird komplett von der Pfanne umhüllt. Quelle: Niethard F, Pfeil J, Biberthaler P. Koxarthrose. In: Biberthaler P, Niethard F, Pfeil J, Hrsg. Duale Reihe Orthopädie und Unfallchirurgie. 7., überarbeitete Auflage. Stuttgart: Thieme; 2014

3,6 $\pm 2,1$ Punkte auf einer 10Punkte Skala (10 = größter Schmerz).

Zu Studienbeginn erfüllten 52 \% der Teilnehmer die ACR-Kriterien für Hüftarthrose und/oder Kniearthrose und von diesen wiederum 91 Teilnehmer (18\%) die Kriterien zu jedem Untersuchungszeitpunkt. Insgesamt genügten 482 Studienteilnehmer(48\%) nicht den ACR-Kriterien zu Studienbeginn und für 138 Probanden (14\%) galten keine der ACR-Kriterien zu keinem Zeitpunkt. Die Mehrheit der Studienteilnehmer erfüllt aber die ACR-Kriterien zu irgendeinem der Untersuchungszeitpunkte (71\% 95\% KI 6874).

Der mediane Wert für Schmerzen nach dem WOMAC-Score blieb über den 10-Jahreszeitraum mit einem Wert von 23(21-25) stabil und auch die Subskalen für Steifheit und Funktion waren ebenfalls relativ konstant. Nach 10 Jahren hatten $60 \%(n=601)$ hatten eine radiologische Arthrose in einem oder beiden Knien und $51 \%(n=513)$ hatten eine radiologische Arthrose in einer oder beiden Hüften. Nur 13,5\% der Studienteilnehmer entwickelten nach 10 Jahren keine radiologische Arthrose. Zu einem Ge- 
lenkersatz nach 10 Jahren kam es 110 Fällen und die meisten Gelenkersatzprothesen wurden bei denjenigen mit mehreren betroffenen Gelenken eingesetzt.

FAZIT

Bei Personen mit Hüft- oder Kniebeschwerden und Verdacht auf Arthrose blieb der symptomatische Verlauf innerhalb der untersuchten Studienpopulation relativ stabil, obwohl Einzelwerte schwankten. Der radiologische Befund zeigte einen progressiven Verlauf mit Gelenkersatz, insbesondere bei Patienten mit beidem, sowohl Hüft- als auch und Kniegelenksarthrose, schreiben die Autoren.

Richard Kessing, Zeiskam 